\title{
7-oxygenated Derivatives of Dehydroepiandrosterone and Obesity
}

\author{
Sedláčková B., Dušátková L., Zamrazilová H., Matucha P., Bičíková M., \\ Stárka L. \\ Institute of Endocrinology, Prague, Czech Republic \\ Received October 25, 2011; Accepted April 11, 2012.
}

Key words: 7-hydroxy-dehydroepiandrosterone - 7-oxo-dehydroepiandrosterone Adiposity - Body mass index - Anthropometric parameters

\begin{abstract}
: 7-hydroxy/oxo derivatives of dehydroepiandrosterone are potential regulators of the local cortisol activity due to their competition in the cortisolcortisone balance mediated by 11ß-hydroxysteroid dehydrogenase. 7-hydroxydehydroepiandrosterone is marketed as anti-obesity medication, though no clinical study aimed at the benefit of administering 7-oxygenated derivatives of dehydroepiandrosterone has appeared until now. We tried to show whether there exist differences in levels of circulating 7-hydroxy/oxo-dehydroepiandrosterone derivatives between lean and obese boys and girls. From a cohort of adolescents investigated within the frame of anti-obesity programme 10 obese boys and 10 obese girls were compared with age-matched lean boys and girls in their anthropometric data, and concentrations of both epimers of 7-hydroxydehydroepiandrosterone and 7-oxo-dehydroepiandrosterone were determined by the RIA method. The basal levels of $7 \alpha$-hydroxy-dehydroepiandrosterone were significantly higher in obese boys than in lean boys but not in girls. The association was found for anthropometric parameters and $7 \alpha$-hydroxy-dehydroepiandrosterone, however again only in boys and not in girls. Higher levels of $7 \alpha$-hydroxydehydroepiandrosterone its positive association with anthropometric data in obese boys may serve as a sign that, at least in boys, 7-oxygenated 5-ene-steroids may take part in regulating the hormonal signal for fat formation or distribution.
\end{abstract}

This study was supported by grants: NS/9831-4 of the Internal Grant Agency of the Ministry of Health of the Czech Republic, CZ0123 from Norway through the Norwegian Financial Mechanisms, and NT 12342-5/2011 of the Ministry of Education, Youth and Sports of the Czech Republic.

Mailing Address: Prof. RNDr. Luboslav Stárka, MD., DSc., Institute of Endocrinology, Národní 8, 11694 Prague 1, Czech Republic; Phone: +420 224905 344; Fax: +420 224905 325; e-mail: Istarka@endo.cz

(C) Charles University in Prague - Karolinum Press, Prague 2012 


\section{Introduction}

$7 \alpha$-hydroxy-dehydroepiandrosterone $(7 \alpha-\mathrm{OH}$-DHEA) was first isolated and identified by Okada et al. (1959) in the urine of a patient with adrenal carcinoma and, soon thereafter (Stárka, 1961; Stárka et al., 1962), in the urine of normal men and women. 7-hydroxylation in rat liver homogenate microsomes was characterized (Stárka and Kutová, 1962) and the 7-hydroxylation of dehydroepiandrosterone was discovered in various tissues, including of the brain (Akwa et al., 1992, 1993; Doostzadeh and Morfin, 1996; Doostzadeh et al., 1997; Rose et al., 1997). Numerous authors paid attention to the presence and role of 7-oxygenated dehydroepiandrosterone derivatives in the brain (Morfin and Stárka, 2001).

For a long time, 7-oxygenated derivatives of dehydroepiandrosterone were considered as degradation metabolites without any metabolic function. However, later on it has been established that at the cellular level, irreversible $7 \alpha$-hydroxylation of 3ß-hydroxy-5-ene steroids produces derivatives, which are believed to be responsible for at least some immunity-promoting and antiglucocorticoid effects (Morfin and Courchay, 1994; Hampl et al., 2000; Morfin et al., 2000; Morfin, 2002; Auci et al., 2009). As "neuroprotective steroids" and immunity promoters in the brain, $7 \alpha$-hydroxy-steroids could contribute to the panels of cellular protection and defense (Morfin and Stárka, 2001; Dušková et al., 2011).

One of the potential ways by which 7-oxygenated dehydroepiandrosterone derivatives exert their protective effects was proposed by several authors (Muller et al., 2006; Hennebert et al., 2007a, b, 2009; Nashev et al., 2007). They documented that these metabolites are substrates for 11ß-hydroxysteroid dehydrogenase type 1 and that they can compete with the transformation of cortisol to cortisone and vice versa. 7-hydroxy-dehydroepiandrosterone has been recently introduced to the market with supplements as an anti-obesity drug (www.dietspotlight.com/lean -xtreme-review/). The idea is based on use of 7-hydroxy-derivatives of DHEA as cortisol regulators and is near to the concept of their function as ergosteroids (Lardy et al., 1995). However, there are only few studies confirming anti-obesitogenic effects of these supplements in humans and only one study, which only marginally deals with effect of transdermally administered 7-oxo-dehydroepiandrosterone (7-oxo-DHEA) on lipid metabolism (Šulcová et al., 2000).

Here we try to show, how the circulating concentrations of dehydroepiandrosterone 7-hydroxy/oxo-derivatives correlate with anthropometric parameters and body composition in teenagers.

\section{Material and Methods}

Groups

Study participants (20 girls and 20 boys) were recruited from the COPAT project (Childhood Obesity Prevalence and Treatment), which had focused on epidemiological (stratified random selection) and intervention (overweight/obesity group) study in Czech adolescents. Age-matched groups of lean (BMI-SDS $<-0.64$ 
or $\mathrm{BMI}<25^{\text {th }}$ percentile) and obese (BMI-SDS $>3.0$ or BMI $>99^{\text {th }}$ percentile) subjects of both sexes in the age of 13.0-17.9 years were studied. Their general anthropometric characterization is shown in Table 1. In the female group 14 girls had regular menstrual cycle, 4 irregular cycle and 2 girls did not report about their menses. Only one girl used hormonal anticonception. Two girls and 4 boys fulfilled the requirements of the criteria of metabolic syndrome according to The International Diabetes Federation (IDF). This difference was not significant. Only two obese girls had glycemia over $5.6 \mathrm{mmol} / \mathrm{l}$ in the range of disturbed glucose tolerance. The values of analytes important for glucose control as glycemia (mean 5.04 vs. $5.025 \mathrm{mmol} / \mathrm{l})$, insulin (15.49 vs. $10.15 \mathrm{mlU} / \mathrm{l})$, C-peptide (0.90 vs. $0.68 \mu \mathrm{g} / \mathrm{l})$ and HOMA-index (3.29 vs. 2.11) for girls and boys did not differ significantly.

The current study was approved by the Ethics Committee of the Institute of Endocrinology and was performed in accordance with the Helsinki Declaration. All participants and their parent(s) provided written informed consent with the original COPAT project. Only those subjects who had agreed that data and blood samples could be used and analysed in subsequent research projects of the Institute of Endocrinology were included in the current pilot study.

\section{Anthropometric characterisation}

Body height was measured with a stadiometer (precision $0.1 \mathrm{~cm}$ ), body weight was measured by Tanita BC-418 MA (precision $0.1 \mathrm{~kg}$ ). The body mass index (BMI) was calculated as the weight in kilograms divided by the square of the height in meters. The percentage of total fat was measured by bioelectric impedance - Tanita BC-418 MA, the percentage of trunk fat (TF) and the degree of visceral fat enlargement (VF) by the Tanita AB-140 ViScan (both Tanita Corporation, Tokyo, Japan). Body circumferences were measured with a tape measure (precision $0.1 \mathrm{~cm}$ ) in a horizontal level; waist circumference midway between the upper iliac crest and the lower rib, abdominal circumference at the level of umbilicus and hip circumference over the maximum buttocks diameter. Sagittal abdominal diameter (SAD) was measured with a pelvimeter at the level L4/5 in a horizontal level.

Skinfolds (biceps, triceps, subscapular, suprailiac) were measured with Best calliper.

\section{Steroid analysis}

Peripheral blood samples were taken from the cubital vein after 12 hours of fasting between 7.00-9.00 a.m. BD Vacutainer $(\circledR$ Plus plastic serum tubes with the silicone coated (Becton Dickinson Vacutainer Systems, UK) were used. Code-identified plasma samples were immediately put in deep frozen boxes (minus 80 degrees Celsius) after blood withdrawal or laboratory analyses to prevent degradation of this biological material. $7 \alpha-$ and $7 \mathrm{~B}-\mathrm{OH}-\mathrm{DHEA}$ were measured in plasma by radioimmunoassays using polyclonal rabbit antisera against 7-OH-DHEA-19-(Ocarboxymethyl)oxime conjugates, with bovine serum albumin and radioiodinated homologous derivatives with tyrosine methyl ester as tracers (Lapčík et al., 1998, 


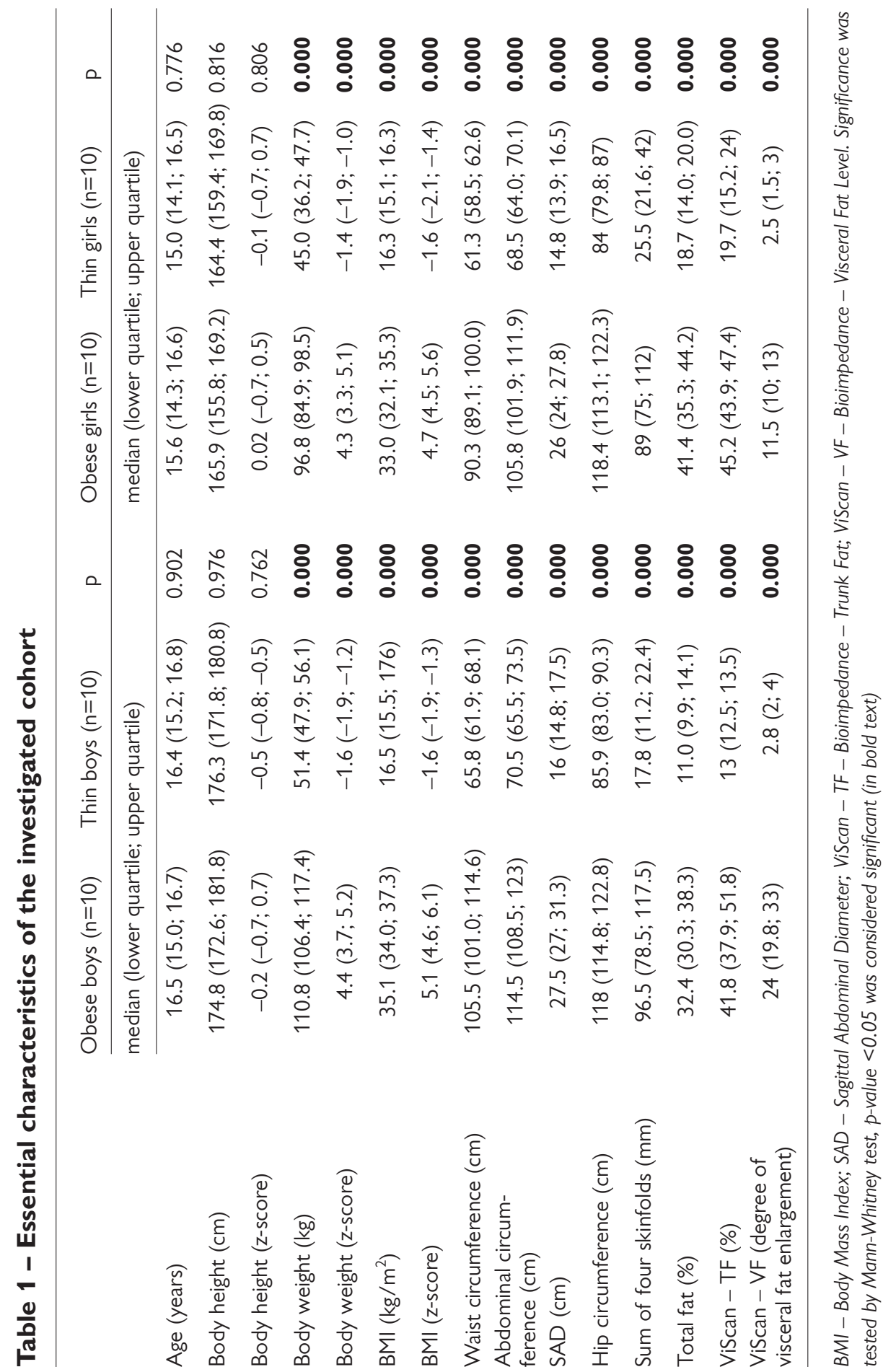


1999). 7-oxo-DHEA was determined by the RIA method according to Kazihnitková et al. (2007). Intra- and interassay coefficients of variation were $6.7 \%$ and $10.0 \%$ for $7 \alpha-\mathrm{OH}$-DHEA, and $7.1 \%$ and $10.6 \%$ for $7 \beta-O H$-DHEA, respectively; the detection limits were 1.06 and $0.95 \mathrm{pg} /$ tube. The cross-reactivity of the antisera with chemically related steroids present in the material analyzed was as follows. For $7 \beta-O H-D H E A$ antibody: $7 \alpha-O H-D H E A$ (the opposite epimer), $0.28 \%$; DHEA, $0.18 \%$; androstenedione, $0.096 \%$; testosterone, $0.013 \%$; cross-reactivity with other hormonal steroids such as cortisol, estradiol, progesterone and dihydrotestosterone was negligible. For $7 \alpha-\mathrm{OH}-\mathrm{DHEA}$ antibody: $7 \mathrm{~B}-\mathrm{OH}-\mathrm{DHEA}, 1.16 \%$; DHEA, 1.95\%; androstenedione, $0.38 \%$; pregnenolone and dihydrotestosterone, $0.04 \%$ each; and testosterone, $0.01 \%$. The cross-reactivity with other hormonal steroids such as cortisol, estradiol, and progesterone was negligible.

\section{Statistical analysis}

Due to non-Gaussian data distribution, the median, lower and upper quartiles were used to describe data. Differences between investigated groups were determined by Mann-Whitney test. The relationships between compared methods were evaluated using non-parametric correlation analysis (the Spearman rank correlation coefficient). The data analysis was performed using the statistical software Statgraphics Plus 5.0 from Statpoint, Inc. (Warrenton, Virginia, USA) and NCSS 2007 (Kaysville, Utah, USA).

\section{Results}

Table 2 shows the principal anthropometric data and serum concentrations of 7-oxygenated DHEA derivatives. Besides anthropometric data a significant difference between obese and lean group was found only for $7 \alpha-\mathrm{OH}-\mathrm{DHEA}$ and only in boys. The basal levels of $7 \alpha-\mathrm{OH}$-DHEA were significantly higher in obese boys than in lean boys.

The association was found for anthropometric parameters (body weight, BMI, body circumferences, SAD, sum of four skinfolds, total fat percentage, TF and VF) and for $7 \alpha$-hydroxydehydroepiandrosterone, however again only in boys and not in girls. In girls there is a significant correlation between body composition (hip circumference, TF, VF) and 7-oxo-DHEA concentration (Table 3).

\section{Discussion}

There are many different theories about the underlying cause of obesity and fat retention and dozens of different ways to try to correct it. Some cortisol regulators based on 7-oxygenated DHEA derivatives are currently marketed as anti-obesity medication.

Dehydroepiandrosterone (DHEA) is hydroxylated to position $7 \alpha$ by the cytochrome P450 7B1 (CYP7B1) in the human brain and liver. This produces $7 \alpha-\mathrm{OH}$-DHEA that is a substrate for $11 \beta$-hydroxysteroid dehydrogenase type 1 


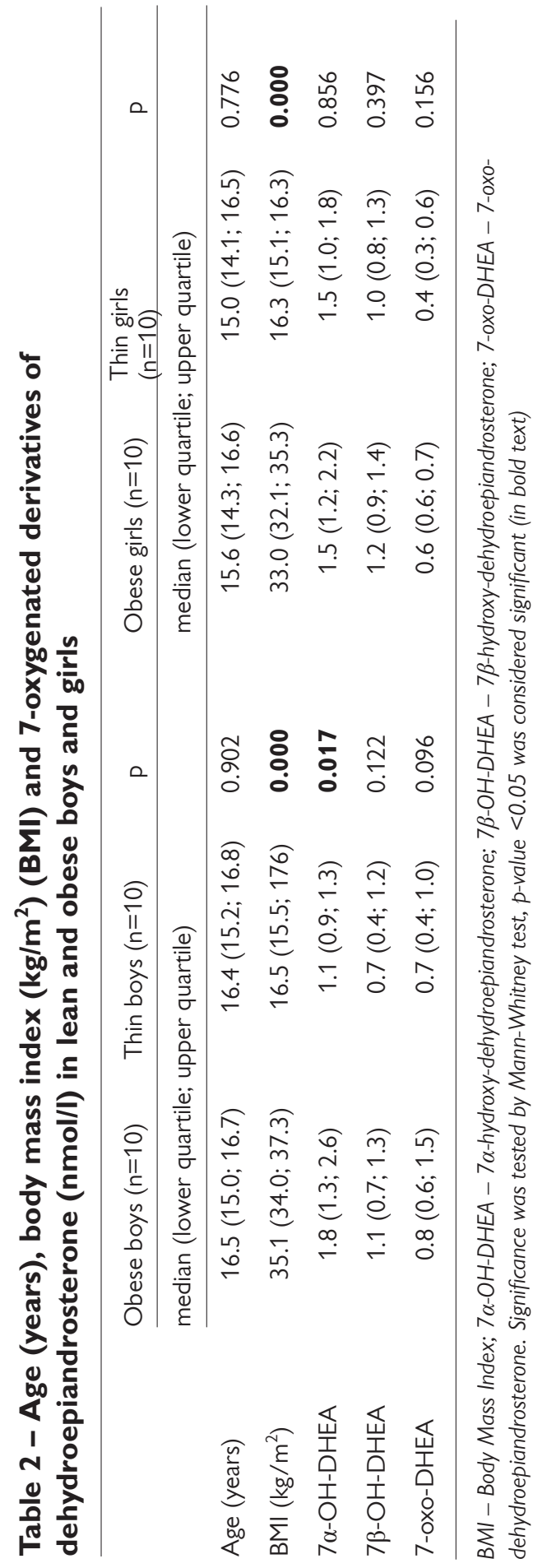

(11 $\beta$-HSD1), which exists in the same tissues and carries out the inter-conversion of $7 \alpha$ - and $7 \beta$-OH-DHEA through a 7-oxointermediary. Since the role of $11 \beta$-HSD1 is to transform the inactive cortisone into active cortisol, its competitive inhibition by $7 \alpha-\mathrm{OH}-\mathrm{DHEA}$ may support the paradigm of native antiglucocorticoid arising from DHEA (Hampl et al., 2000; Morfin et al., 2000; Morfin, 2002; Hennebert et al., 2007a, b). The metabolic abnormalities found associated with high blood glucocorticoid levels include insulin-resistance, visceral obesity, hypertension, dyslipidaemia and an increased risk of cardiovascular diseases (Anagnostis et al., 2009; Morton, 2010). Therefore, our objective was to investigate whether the concentration of 7-oxo/oxy derivatives of DHEA are associated with anthropometric and metabolic parameters, which are known to be dependent at least partially on glucocorticoid activity (as adiposity and fat distribution, lipid spectrum, or insulin resistance). These findings may support the previously proposed native anti-glucocorticoid paradigm and suggest that $7 \alpha-\mathrm{OH}$ DHEA production is a key for the fine tuning of glucocorticoid levels in tissues.

Our pilot study with limited number of individuals under investigation shows some association of higher $7 \alpha-\mathrm{OH}$-DHEA levels with anthropometric data in obese 


\section{Table 3 - Spearman's correlations between circulating 7-oxygenated derivatives of DHEA and anthropometric parameters and body composition}

\begin{tabular}{|c|c|c|c|c|c|c|c|}
\hline \multirow{4}{*}{ Height (cm) } & \multirow[b]{3}{*}{ r } & \multicolumn{2}{|c|}{$7 \alpha-\mathrm{OH}-\mathrm{DHEA}$} & \multicolumn{2}{|c|}{$7 \beta-O H-D H E A$} & \multicolumn{2}{|c|}{ 7-oxo-DHEA } \\
\hline & & boys & girls & boys & girls & boys & girls \\
\hline & & -0.184 & -0.020 & -0.470 & 0.039 & -0.055 & 0.126 \\
\hline & $P$ & 0.451 & 0.935 & 0.042 & 0.875 & 0.823 & 0.607 \\
\hline \multirow{2}{*}{ Body weight (kg) } & r & 0.675 & 0.090 & 0.212 & 0.154 & 0.233 & 0.369 \\
\hline & $\mathrm{P}$ & 0.002 & 0.175 & 0.383 & 0.528 & 0.337 & 0.120 \\
\hline \multirow{2}{*}{ BMI $\left(\mathrm{kg} / \mathrm{m}^{2}\right)$} & r & 0.696 & 0.206 & 0.326 & 0.272 & 0.222 & 0.385 \\
\hline & $P$ & 0.000 & 0.397 & 0.173 & 0.260 & 0.359 & 0.103 \\
\hline \multirow{2}{*}{ Waist circumference (cm) } & r & 0.603 & 0.107 & 0.146 & 0.119 & 0.321 & 0.277 \\
\hline & $P$ & 0.006 & 0.663 & 0.551 & 0.628 & 0.180 & 0.251 \\
\hline \multirow{2}{*}{ Abdominal circumference $(\mathrm{cm})$} & r & 0.650 & 0.225 & 0.253 & 0.313 & 0.175 & 0.366 \\
\hline & $P$ & 0.003 & 0.355 & 0.295 & 0.193 & 0.743 & 0.124 \\
\hline \multirow{2}{*}{$\mathrm{SAD}(\mathrm{cm})$} & r & 0.547 & 0.045 & 0.178 & -0.091 & 0.187 & 0.446 \\
\hline & $\mathrm{P}$ & 0.019 & 0.861 & 0.480 & 0.717 & 0.456 & 0.064 \\
\hline \multirow{2}{*}{ Hip circumference (cm) } & r & 0.701 & 0.278 & 0.255 & 0.307 & 0.141 & 0.485 \\
\hline & $P$ & 0.000 & 0.250 & 0.292 & 0.202 & 0.565 & 0.035 \\
\hline \multirow{2}{*}{ Subscapular skinfold (mm) } & r & 0.774 & -0.051 & 0.506 & 0.045 & 0.291 & 0.189 \\
\hline & $P$ & 0.000 & 0.834 & 0.027 & 0.856 & 0.227 & 0.438 \\
\hline \multirow{2}{*}{ Sum of four skinfolds (mm) } & r & 0.743 & 0.183 & 0.407 & 0.248 & 0.168 & 0.391 \\
\hline & $P$ & 0.000 & 0.453 & 0.084 & 0.306 & 0.495 & 0.100 \\
\hline \multirow{2}{*}{ Total fat (\%) } & r & 0.639 & 0.176 & 0.449 & 0.228 & 0.006 & 0.403 \\
\hline & $P$ & 0.004 & 0.484 & 0.062 & 0.363 & 0.827 & 0.097 \\
\hline \multirow{2}{*}{ ViScan - TF (\%) } & r & 0.589 & 0.263 & 0.280 & 0.241 & 0.412 & 0.627 \\
\hline & $P$ & 0.016 & 0.385 & 0.294 & 0.428 & 0.112 & 0.022 \\
\hline \multirow{2}{*}{ ViScan - VF (fat level) } & r & 0.526 & 0.336 & 0.121 & 0.241 & 0.285 & 0.591 \\
\hline & $P$ & 0.036 & 0.261 & 0.657 & 0.428 & 0.284 & 0.033 \\
\hline
\end{tabular}

BMI - Body Mass Index; SAD - Sagittal Abdominal Diameter; ViScan - TF - Bioimpedance - Trunk Fat; ViScan - VF - Bioimpedance - Visceral Fat Level; $7 \alpha$-OH-DHEA - 7 $\alpha$-hydroxy-dehydroepiandrosterone; $7 \beta$-OH-DHEA

- 7 $\beta$-hydroxy-dehydroepiandrosterone; 7-oxo-DHEA - 7-oxo-dehydroepiandrosterone; $r$ - regression coefficient; $p-p$-value. Data significant at $p<0.05$ are in bold text

boys, not in girls. We have no explanation for this difference between the sexes, gender differences in DHEA metabolism or lower level of 7-oxygenated derivatives 
of DHEA in girls might play some role as well as lower incidence of metabolic syndrome in girls than in boys. The increase of $7 \alpha-\mathrm{OH}$-DHEA may be a result of the increased production and conversion of 7-oxo-DHEA as a substrate for $11 \beta$-hydroxysteroid dehydrogenase type 1 (11 $\beta$-HSD1) and thus decreasing the concentration of cortisol on account of cortisone. Inhibitors of $11 \beta$-HSD1 might represent a promising therapeutic target in obesity and metabolic syndrome.

To answer the question whether endogenous competitive substrates for $11 \beta$-HSD1 as 7-oxydenated derivatives of DHEA and perhaps also of pregnenolone are really involved in fat accumulation more investigations in this field are needed.

\section{Conclusion}

Higher levels of $7 \alpha$-hydroxy-dehydroepiandrosterone and its positive association with anthropometric data in obese boys may serve as a sign that, at least in boys, 7-oxygenated 5-ene-steroids may take part in regulating the hormonal signal for fat formation or distribution.

\section{References}

Akwa, Y., Morfin, R. F., Robel, P., Baulieu, E. E. (1992) Neurosteroid metabolism. 7 alpha-hydroxylation of dehydroepiandrosterone and pregnenolone by rat brain microsomes. Biochem. J. 288, 959-964.

Akwa, Y., Sananès, N., Gouézou, M., Robel, P., Baulieu, E. E., Le Goascogne, C. (1993) Astrocytes and neurosteroids: metabolism of pregnenolone and dehydroepiandrosterone. Regulation by cell density. J. Cell Biol. 121, 135-143.

Anagnostis, P., Athyros, V. G., Tziomalos, K., Karagiannis, A., Mikhailidis, D. P. (2009) Clinical review: The pathogenetic role of cortisol in the metabolic syndrome: a hypothesis. J. Clin. Endocrinol. Metab. 94, 2692-2701.

Auci, D. L., Reading, C. L., Frincke, J. M. (2009) 7-hydroxy androstene steroids and a novel synthetic analogue with reduced side effects as a potential agent to treat autoimmune diseases. Autoimmun. Rev. 8, 369-372.

Doostzadeh, J., Morfin, R. (1996) Studies of the enzyme complex responsible for pregnenolone and dehydroepiandrosterone7 alpha-hydroxylation in mouse tissues. Steroids 61, 613-620.

Doostzadeh, J., Cotillon, A. C., Morfin, R. (1997) Dehydroepiandrosterone 7alpha- and 7beta-hydroxylation in mouse brain microsomes. Effects of cytochrome P450 inhibitors and structure-specific inhibition by steroid hormones. J. Neuroendocrinol. 9, 923-928.

Dušková, M., Šimůnková, K., Hill, M., Stárka, L. (2011) 7-hydroxylated derivatives of dehydroepiandrosterone as possibly related to menstrual mood change in healthy women. Endocr. Regul. 45, 131-137.

Hampl, R., Lapčík, O., Hill, M., Klak, J., Kasal, A., Nováček, A., Šterzl, I., Šterzl, J., Stárka, L. (2000)

7-hydroxydehydroepiandrosterone - a natural antiglucocorticoid and a candidate for steroid replacement therapy? Physiol. Res. 49, 107-112 (Suppl. 1).

Hennebert, O., Le Mée, S., Pernelle, C., Morfin, R. (2007a) 5Alpha-androstane-3beta,7alpha,17beta-triol and 5 alpha-androstane-3beta,7beta,17beta-triol as substrates for the human 11 beta-hydroxysteroid dehydrogenase type 1. Steroids 72, 855-864.

Hennebert, O., Chalbot, S., Alran, S., Morfin, R. (2007b) Dehydroepiandrosterone 7alpha-hydroxylation in human tissues: possible interference with type 11beta-hydroxysteroid dehydrogenase-mediated processes. J. Steroid Biochem. Mol. Biol. 104, 326-333. 
Hennebert, O., Montes, M., Favre-Reguillon, A., Chermette, H., Ferroud, C., Morfin, R. (2009) Epimerase activity of the human 11beta-hydroxysteroid dehydrogenase type 1 on 7-hydroxylated C19-steroids. J. Steroid Biochem. Mol. Biol. 114, 57-63.

Kazihnitková, H., Zamrazilová, L., Hill, M., Lapčík, O., Pouzar, V., Hampl, R. (2007) A novel radioimmunoassay of 7-oxo-DHEA and its physiological levels. Steroids 72, 342-350.

Lapčík, O., Hampl, R., Hill, M., Bičíková, M., Stárka, L. (1998) Immunoassay of 7-hydroxysteroids:

1. Radioimmunoassay of 7ß-hydroxy-dehydroepiandrosterone. J. Steroid Biochem. Mol. Biol. 67, 439-445.

Lapčík, O., Hampl, R., Hill, M., Stárka, L. (1999) Immunoassay of 7-hydroxysteroids: 2. Radioimmunoassay of 7 $\alpha$-hydroxy-dehydroepiandrosterone. J. Steroid Biochem. Mol. Biol. 71, 231-237.

Lardy, H., Partridge, B., Kneer, N., Wei, Y. (1995) Ergosteroids: induction of thermogenic enzymes in liver of rats treated with steroids derived from dehydroepiandrosterone. Proc. Natl. Acad. Sci. USA 92, 6617-6619.

Morfin, R. (2002) Involvement of steroids and cytochromes P(450) species in the triggering of immune defenses. J. Steroid Biochem. Mol. Biol. 80, 273-290.

Morfin, R., Courchay, G. (1994) Pregnenolone and dehydroepiandrosterone as precursors of native 7-hydroxylated metabolites which increase the immune response in mice. J. Steroid Biochem. Mol. Biol. 50, 91-100.

Morfin, R., Stárka, L. (2001) Neurosteroid 7-hydroxylation products in the brain. Int. Rev. Neurobiol. 46, 79-95.

Morfin, R., Lafaye, P., Cotillon, A. C., Nato, F., Chmielewski, V., Pompon, D. (2000) 7alpha-hydroxydehydroepiandrosterone and immune response. Ann. N. Y. Acad. Sci. 917, 971-982.

Morton, N. M. (2010) Obesity and corticosteroids: 11beta-hydroxysteroid type 1 as a cause and therapeutic target in metabolic disease. Mol. Cell. Endocrinol. 316, 154-164.

Muller, C., Pompon, D., Urban, P., Morfin, R. (2006) Inter-conversion of 7alpha- and 7beta-hydroxydehydroepiandrosterone by the human 11beta-hydroxysteroid dehydrogenase type 1. J. Steroid Biochem. Mol. Biol. 99, 215-222.

Nashev, L. G., Chandsawangbhuwana, C., Balazs, Z., Atanasov, A. G., Dick, B., Frey, F. J., Baker, M. E., Odermatt, A. (2007) Hexose-6-phosphate dehydrogenase modulates 11 beta-hydroxysteroid dehydrogenase type 1-dependent metabolism of 7-keto- and 7beta-hydroxy-neurosteroids. PLoS One $\mathbf{2}$, e561.

Okada, M., Fukushima, D. K., Gallagher, T. F. (1959) Isolation and characterization of 3 beta-hydroxy-delta 5-steroids in adrenal carcinoma. J. Biol. Chem. 234, 1688-1692.

Rose, K. A., Stapleton, G., Dott, K., Kieny, M. P., Best, R., Schwarz, M., Russell, D. W., Björkhem, I., Seckl, J., Lathe, R. (1997) Cyp7b, a novel brain cytochrome P450, catalyzes the synthesis of neurosteroids 7alphahydroxy dehydroepiandrosterone and 7alpha-hydroxy pregnenolone. Proc. Natl. Acad. Sci. USA 94, 4925-4930.

Stárka, L. (1961) Reaktion der Steroide mit tert-Butylperbenzoat I. Über die 7-acyloxylierung $\Delta^{5}$-ungesättigter Steroide. Collect. Czech. Chem. Commun. 26, 2452-2456.

Stárka, L., Kutová, J. (1962) 7-hydroxylation of dehydroepiandrosterone by rat-liver homogenate. Biochim. Biophys. Acta 56, 76-82.

Stárka, L., Šulcová, J., Šilink, K. (1962) Die Harnausscheidung des 7-Hydroxydehydroepi- androsteronsulfates. Clin. Chim. Acta 7, 309-316.

Šulcová, J., Hill, M., Hampl, R., Mašek, Z., Nováček, A., Češka, R., Stárka, L. (2000) Effects of transdermal application of DHEA on the levels of steroids, gonadotropins and lipids in men. Physiol. Res. 49, 685-693. 